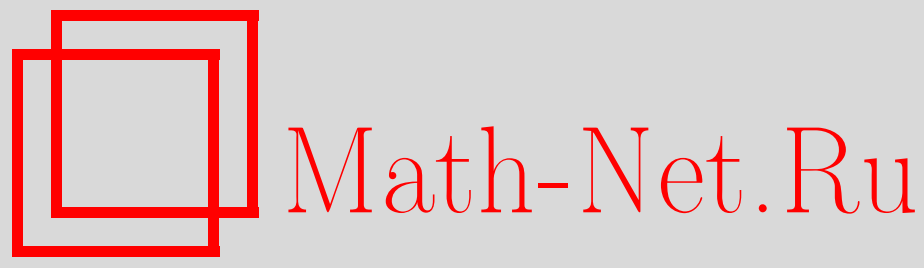

В. Г. Михайлов, Предельная теорема пуассоновского типа для числа пар почти полностью совпавших цепочек, Теория вероятн. и ее примен., 2008, том 53, выпуск 1, 59-71

DOI: https://doi.org/10.4213/tvp319

Использование Общероссийского математического портала MathNet.Ru подразумевает, что вы прочитали и согласны с пользовательским соглашением

http://www . mathnet.ru/rus/agreement

Параметры загрузки:

IP : 18.209 .158 .208

26 апреля 2023 г., 10:53:10

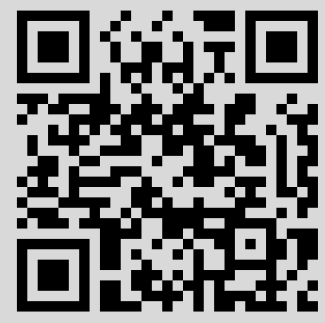


(c) 2008 г.

МИХАЙЛОВ В. Г.*

\title{
ПРЕДЕЛЬНАЯ ТЕОРЕМА ПУАССОНОВСКОГО ТИПА ДЛЯ ЧИСЛА ПАР ПОЧТИ ПОЛНОСТЬЮ СОВПАВШИХ ЦЕПОЧЕК ${ }^{1)}$
}

\begin{abstract}
Рассматриваются две последовательности $X_{1}, \ldots, X_{m}$ и $Y_{1}, \ldots$, $Y_{n}$ из независимых одинаково распределенных внутри каждой последовательности случайных величин, принимающих значения из множества $\{1,2, \ldots\}$. Изучается распределение числа $N_{d}$ таких пар $s$-цепочек $\left(\bar{X}_{i}, \bar{Y}_{j}\right)$, где $\bar{X}_{i}=\left(X_{i}, \ldots, X_{i+s-1}\right), \bar{Y}_{j}=$ $\left(Y_{j}, \ldots, Y_{j+s-1}\right)$, в которых $s$-цепочки $\bar{X}_{i}$ и $\bar{Y}_{j}$ различаются относительно небольшим числом элементов $d$. Показано, что в схеме серий при $m, n, s \rightarrow \infty, d=o(s / \ln s)$ и таком изменении распределений последовательностей, что вероятность $\mathbf{P}\left\{X_{i}=Y_{j}\right\}$ и среднее $\mathrm{E} N_{d}$ имеют пределы, распределение случайной величины $N_{d}$ сходится к сложному пуассоновскому распределению. Значение параметра $d$ учитывается лишь при согласовании параметров схемы при переходе к пределу, а на вид предельного распределения оно не влияет. Это предельное распределение точно такое же, какое имеет в аналогичном случае число пар $\left(\bar{X}_{i}, \bar{Y}_{j}\right)$, в которых $\bar{X}_{i}=\bar{Y}_{j}$.
\end{abstract}

Ключевые слова и фразы: s-цепочки, совпадения цепочек, совпадения цепочек с нарушениями, предельная теорема Пуассона, сложное распределение Пуассона, метод Чена-Стейна.

1. Формулировка и обсуждение теоремы. Пусть $X=\left(X_{1}, \ldots\right.$, $\left.X_{m}\right)$ и $Y=\left(Y_{1}, \ldots, Y_{n}\right)$ - две последовательности независимых одинаково (внутри каждой последовательности) распределенных случайных величин, принимающих значения в множестве $\{1,2, \ldots\}$,

$$
\begin{aligned}
p_{k} & =\mathbf{P}\left\{X_{1}=k\right\}, \quad q_{k}=\mathbf{P}\left\{Y_{1}=k\right\}, \quad k=1,2, \ldots, \\
p & =\max _{k}\left(p_{k} I\left\{q_{k}>0\right\}\right), \quad q=\max _{k}\left(q_{k} I\left\{p_{k}>0\right\}\right)
\end{aligned}
$$

* Математический институт им. В. А. Стеклова РАН, ул. Губкина, 8, 119991 Москва, ГСП-1, Россия.

1) Работа выполнена при поддержке программы РАН «Современные проблемы теоретической математики», Российского фонда фундаментальных исследований (гранты 02-01-00266 и 05-01-00035) и программы «Ведушие научные школы» (грант НШ-1758.2003.1). 
(здесь и далее выражение $I\{\ldots\}$ обозначает индикатор множества или случайного события),

$$
r=\max _{k} p_{k} q_{k}, \quad R=\mathbf{P}\left\{X_{1}=Y_{1}\right\}=\sum_{k=1}^{\infty} p_{k} q_{k} .
$$

Мы разместим случайные величины $X_{1}, \ldots, X_{m}$ и $Y_{1}, \ldots, Y_{n}$ в вершины дискретных окружностей длин $m$ и $n$ соответственно и, задав натуральные числа $c, s, 1 \leqslant c \leqslant s$, рассмотрим события

$$
H_{i, j}(s, c)=\left\{\sum_{k=0}^{s-1} I\left\{X_{i+k}=Y_{j+k}\right\}=c\right\} .
$$

В соответствии с порядком вершин на окружностях здесь считается, что $X_{m+i}=X_{i}, Y_{n+i}=Y_{i}$ при всех $i=1,2, \ldots$.

В работе [7] событие $H_{i, j}(s, c)$ было названо $(s, c)$-совпадением цепочек $\bar{X}_{i}=\left(X_{i}, \ldots, X_{i+s-1}\right)$ и $\bar{Y}_{j}=\left(Y_{j}, \ldots, Y_{j+s-1}\right)$. Его называют также совпадением (цепочек $\bar{X}_{i}$ и $\bar{Y}_{j}$ ) с дефектом $d=s-c$. При $c=s$ это обычное совпадение цепочек. Число $(s, c)$-совпадений (совпадений с дефектом $d=s-c)$ выражается формулой

$$
N_{d}=\sum_{i=1}^{m} \sum_{j=1}^{n} H_{i, j}(s, c) .
$$

Теорема 1. Пусть параметры схемь $m, n, s$ стремятся $\kappa$ бесконечности, а вероятности $p_{k}, q_{k}$ меняются так, что

$$
\begin{aligned}
& \Lambda=m n C_{s}^{c} R^{c}(1-R)^{s-c+1} \longrightarrow \lambda, \\
& 0<\lambda<\infty, \quad R \rightarrow \rho, \quad 0 \leqslant \rho<1,
\end{aligned}
$$

причем

$$
\begin{gathered}
\frac{d \ln s}{s} \rightarrow 0, \\
\frac{s}{m}+\frac{s}{n} \rightarrow 0, \\
s C_{s}^{d}\left(m p^{c}+n q^{c}+\frac{s}{(1-R)^{c-s}}\left(\frac{r}{R}\right)^{c}\right) \longrightarrow 0 .
\end{gathered}
$$

Тогда распределение случайной величинь $N_{d}$ сходится $\kappa$ сложному пуассоновскому распределению с производящей функиией

$$
\exp \left\{\frac{\lambda(z-1)}{1-\rho z}\right\} \text {. }
$$

3 а м е ч а н и е 1 . Из условий теоремы следует, что $d=s-c=$ $o(s)$. Таким образом, в теореме 1 речь идет о почти полностью совпавших $s$-цепочках.

3 а м е ч а н и е 2. Условие (1.2) может быть ослаблено. Достаточно предположить, что

$$
\frac{d}{s}\left(1+\frac{\ln s}{|\ln R|}\right) \longrightarrow 0 .
$$


З а м е ч а н и е 3 . В ходе доказательства вырисовывается следующая картина асимптотического поведения $(s, c)$-совпадений цепочек. Эти совпадения возникают сериями случайной длины, число серий имеет в пределе распределение Пуассона с параметром $\lambda$, а длины серий асимптотически независимы и имеют в пределе геометрическое распределение с параметром $\rho$. Предельная теорема Пуассона для числа серий была получена ранее (см. [1]-[3]). Новизна нашего результата заключается в нахождении асимптотического распределения длин серий.

3 а м е ч а н и е 4. Среди величин $N_{d}$ особый интерес представляет случайная величина $N_{0}$ - число пар одинаковых $s$-цепочек в последовательностях $X$ и $Y$. Она изучалась многими авторами (например, в [1]-[6]). В случае $c=s$ наша теорема дает такие же условия сходимости к указанному выше предельному распределению, как и следствие 1 работы [6].

3 а м е ч а н и е 5 . Теорема 1 показывает, что при $\rho \rightarrow 0$ в качестве предельного распределения для $N_{d}$ выступает распределение Пуассона. Для величины $N_{0}$ предельная теорема Пуассона при разных условиях доказывалась в работах [1]-[6].

3 а м е ч а н и е 6. Свойства случайных величин $N_{d}$ при $c<s$ в случае фиксированных значений параметров $s, c$ изучались в работе [7], где была доказана сходимость распределения $(d+1)$-мерного случайного вектора $\bar{N}_{d}=\left(N_{0}, N_{1}, \ldots, N_{d}\right)$ к распределению вектора

$$
\left(\sum_{k=c}^{l}(l-k+1) \pi_{k}, l=c, \ldots, s\right),
$$

где $\pi_{k}, k=c, \ldots, s$, - независимые случайные величины, распределенные по закону Пуассона с параметрами $\lambda_{k}=C_{k-2}^{c-2} \lambda, k=c, \ldots, s$, соответственно, а $\lambda=\lim m n R^{c}$. Одномерные компоненты этого распределения принципиально отличаются от предельного распределения в нашем случаe.

З а м е ч ан и е 7. Утверждение теоремы 1 справедливо и для $(s, c)$-совпадений цепочек из обычных (не замкнутых в циклы) последовательностей случайных величин. Возникающие в этом случае эффекты, связанные со спецификой свойств совпадений цепочек, расположенных в началах и концах последовательностей, лишь несколько усложняют рассуждения.

2. Доказательство теоремы. Первый этап доказательства разобраться в общей картине асимптотического поведения $(s, c)$-совпадений. Здесь поможет следующее соображение. Вероятность появления $(s, c)$-совпадения на заданных местах последовательностей зависит от числа несовпадений, но не зависит от того, на каких именно местах буквы в цепочках не совпали. Используя этот факт, можно показать, что 
для основной массы $(s, c)$-совпадений расстояние от начала цепочки до места первого несовпадения в цепочках и расстояние от места последнего несовпадения до конца цепочки стремятся к бесконечности. Поэтому, исключив из рассмотрения иные $(s, c)$-совпадения, мы не изменим предельное распределение для их общего числа. Осталось заметить, что $(s, c)$-совпадение с координатами $(i, j)$, начинающееся и заканчивающееся полностью совпавшими участками цепочек, влечет с вероятностью $R$ такое же $(s, c)$-совпадение с координатами $(i+1, j+1)$. Это свойство не зависит от значения параметра $d$, если он мал относительно $s$, и выполнено для полных совпадений цепочек. Поэтому основная масса $(s, c)$-совпадений образует скопления, число совпадений в скоплении имеет в пределе геометрическое распределение, а сами скопления представляют собой редкие слабо зависимые события.

Эта картина полностью аналогична картине, наблюдаемой для полных совпадений цепочек. Предельное поведение числа пар совпавших цепочек хорошо изучено (см., например, [6], [8], [9]).

Приняв в качестве естественной гипотезы эту картину, сгруппируем $(s, c)$-совпадения в серии и попробуем доказать многомерную предельную теорему Пуассона для вектора из чисел серий из заданного количества $(s, c)$-совпадений. Для ее доказательства мы воспользуемся функциональным вариантом метода Чена-Стейна.

3 а м е ч а н и е 8 . Описанный выше прием отсева части $(s, c)$-совпадений использовался ранее в работах [1]-[3].

Перейдем к реализации этого плана. Начнем с отсева неудобных для нас $(s, c)$-совпадений. Зададим натуральное число $g, 1 \leqslant g \leqslant c / 2$, и рассмотрим события

$$
\begin{aligned}
H_{i, j}(s, c, g)=H_{i, j}(s, c) & \bigcap \bigcap_{k=1}^{g}\left\{X_{i+k-1}=Y_{j+k-1}\right\} \\
& \bigcap \bigcap_{k=0}^{g-1}\left\{X_{i+s-g+k}=Y_{j+s-g+k}\right\} .
\end{aligned}
$$

Здесь речь идет о таких $(s, c)$-совпадениях, в которых на первых и последних $g$ местах цепочек соответствующие буквы цепочек совпадают. Поэтому для «несовпадений букв» в цепочках имеется лишь $s-2 g$ возможных позиций. Такие $(s, c)$-совпадения мы будем называть $(s, c, g)$-совпадениями. Их число выражается формулой

$$
N_{d}(g)=\sum_{i=1}^{m} \sum_{j=1}^{n} H_{i, j}(s, c, g) \text {. }
$$

Как и раньше, здесь считается, что $X_{m+i}=X_{i}, Y_{n+i}=Y_{i}$ при всех $i=1,2, \ldots$. 
Лемма 1. Пусть $m, n, s \rightarrow \infty$, вьполнено условие (1.1), а параметр $g$ меняется так, что

$$
g\left(1-c s^{-1}\right) \rightarrow 0
$$

Tozдa

$$
\mathbf{P}\left\{N_{d}=N_{d}(g)\right\} \rightarrow 1
$$

Д о к а з а т е л ь с т в о. Из определений следуют равенства

$$
\begin{gathered}
\mathbf{P}\left\{H_{i, j}(s, c)\right\}=C_{s}^{c} R^{c}(1-R)^{d}, \\
\mathbf{P}\left\{H_{i, j}(s, c, g)\right\}=C_{s-2 g}^{c-2 g} R^{c}(1-R)^{d} .
\end{gathered}
$$

Кроме того, $N_{d} \geqslant N_{d}(g)$. Значит,

$$
\begin{aligned}
\mathbf{P}\left\{N_{d} \neq N_{d}(g)\right\} & \leqslant \mathbf{E} N_{d}-\mathbf{E} N_{d}(g)=\left(C_{s}^{c}-C_{s-2 g}^{c-2 g}\right) m n R^{c}(1-R)^{d} \\
& =\left(1-\frac{(s-2 g)_{d}}{(s)_{d}}\right) \frac{\Lambda}{1-R}
\end{aligned}
$$

(здесь и далее $(s)_{d}=s(s-1) \cdots(s-d+1)$ ).

Заметим, что

$$
\frac{(s-2 g)_{d}}{(s)_{d}}=\left(1-\frac{2 g}{s}\right)\left(1-\frac{2 g}{s-1}\right) \cdots\left(1-\frac{2 g}{c+1}\right),
$$

а при достаточно малых по сравнению с $s$ величинах $d=s-c$ и $g$

$$
1-\frac{(s-2 g)_{d}}{(s)_{d}} \leqslant 2 g \sum_{k=0}^{d-1} \frac{1}{s-k}=O\left(g \frac{d}{c}\right) .
$$

Из условий леммы следует, что правая часть соотношения (2.4) стремится к нулю, а из (1.1), (2.3) и (2.4) вытекает, что $\mathbf{P}\left\{N_{d} \neq N_{d}(g)\right\} \rightarrow 0$. Лемма 1 доказана.

Согласно лемме 1 , при рассматриваемых условиях предельные распределения случайных величин $N_{d}$ и $N_{d}(g)$ совпадают. Поэтому мы перейдем к изучению асимптотических свойств величины $N_{d}(g)$, что делается более просто.

При $k=1,2, \ldots$ введем события

$$
G(i, j, k)=\left\{X_{i-1} \neq Y_{j-1}\right\} \cap \bigcap_{l=0}^{k-1} H_{i+l, j+l}(s, c, g) \cap\left\{X_{i+k+s-1} \neq Y_{j+k+s-1}\right\}
$$

и случайные величины $W(i, j, k)=I\{G(i, j, k)\}$.

Случайная величина $W(i, j, k)$ является индикатором серии из $k$ смежных $(s, c, g)$-совпадений, начинаюшейся с совпадения $H_{i, j}(s, c, g)$. Такая серия не может быть слишком длинной, поскольку она обязательно начинается с $g+k-1$ совпадений букв, а на первых $s$ местах 
должны еще уместиться $s-c$ несовпадений букв. Поэтому $k \leqslant h=$ $c-2 g+1$. Значит, можно написать формулу

$$
N_{d}(g)=\sum_{i=1}^{m} \sum_{j=1}^{n} \sum_{k=1}^{h} k W(i, j, k) .
$$

Далее нам удобно ввести обозначения

$$
\Gamma=\{a=(i, j, k): i \in\{1, \ldots, m\}, j \in\{1, \ldots, n\}, k \in\{1, \ldots, h\}\} .
$$

Тогда сумма (2.6) запишется следующим образом:

$$
N_{d}(g)=\sum_{a \in \Gamma} k W(a) .
$$

Пусть $W$ обозначает набор случайных величин $\{W(a)\}_{\Gamma}$, а $\pi=$ $\{\pi(a)\}_{\Gamma}$ - набор независимых в совокупности случайных величин, имеющих распределения Пуассона с параметрами $\mathbf{E} \pi(a)=\mathbf{E} W(a), a \in \Gamma$.

Рассмотрим расстояние по вариации $\rho\left(\mathbf{P}_{1}, \mathbf{P}_{2}\right)$ между распределениями $\mathbf{P}_{1}$ и $\mathbf{P}_{2}$. Для распределений на дискретном множестве $A$ оно выражается формулой

$$
\rho\left(\mathbf{P}_{1}, \mathbf{P}_{2}\right)=\frac{1}{2} \sum_{a \in A}\left|\mathbf{P}_{1}(a)-\mathbf{P}_{2}(a)\right| .
$$

Распределение случайной величины (случайного набора) $W$ будем обозначать $\mathscr{L}(W)$.

Лемма 2. Пусть $m, n>s=c+d \geqslant c \geqslant 2 g$. Тогда

$$
\rho(\mathscr{L}(W), \mathscr{L}(\pi)) \leqslant S_{1}+S_{2},
$$

zde

$$
\begin{aligned}
& S_{1}=(4 s+3) \Lambda^{2}\left(\frac{1}{m}+\frac{1}{n}\right) \\
& S_{2}=4 s \Lambda\left(\frac{R^{g}}{2(1-R)}+C_{s}^{s-c}\left(m p^{c}+n q^{c}\right)+\frac{4 s C_{s}^{s-c}}{(1-R)^{s-c+1}}\left(\frac{r}{R}\right)^{c}\right) .
\end{aligned}
$$

Д оказательство леммы 2 . Определим множество $\Gamma_{a}^{\text {ind }}$ следующим образом:

$$
\begin{aligned}
& \Gamma_{a}^{\text {ind }}=\left\{a^{\prime}=\left(i^{\prime}, j^{\prime}, k^{\prime}\right) \in \Gamma:\right. i^{\prime}-i \geqslant s+k+1 \text { или } i-i^{\prime} \geqslant s+k^{\prime}+1 \\
&\text { и } \left.j^{\prime}-j \geqslant s+k+1 \text { или } j-j^{\prime} \geqslant s+k^{\prime}+1\right\} .
\end{aligned}
$$

Нетрудно проверить, что случайная величина $W(a)$ не зависит от совокупности случайных величин $\left\{W\left(a^{\prime}\right), a^{\prime} \in \Gamma_{a}^{\text {ind }}\right\}$. Положим

$$
\Gamma_{a}=(\Gamma \backslash\{a\}) \backslash \Gamma_{a}^{\text {ind }} .
$$

Воспользуемся теоремой 10.А книги [12], из которой следует, что $\rho(\mathscr{L}(W), \mathscr{L}(\pi)) \leqslant \sum_{a \in \Gamma} \mathbf{E} W(a)(\mathbf{E} W(a)+\mathbf{E} U(a))+\sum_{a \in \Gamma} \mathbf{E} W(a) U(a)$, 
где

$$
U(a)=\sum_{a^{\prime} \in \Gamma_{a}} W\left(a^{\prime}\right)
$$

Определим множество

$$
\Gamma(r)=\{a=(i, j, k) \in \Gamma: k=r\} .
$$

Чтобы оценить первую сумму в правой части (2.11), заметим, что

$$
\begin{aligned}
\left|\left(\{a\} \cup \Gamma_{a}\right) \cap \Gamma(r)\right| & =\left|\left(\Gamma \backslash \Gamma_{a}^{\text {ind }}\right) \cap \Gamma(r)\right| \\
& \leqslant(m n-(m-2(s+h)-3)(n-2(s+h)-3)) \\
& \leqslant(2(s+h)+3)(m+n) .
\end{aligned}
$$

Из равенства (2.5) при $k \leqslant h$ вытекает формула

$$
\begin{aligned}
& \mathbf{E} W(i, j, k)=\mathbf{P}\{G(i, j, k)\} \\
& \quad=\mathbf{P}\left\{X_{i-1} \neq Y_{j-1}\right\} \mathbf{P}\left\{\bigcap_{l=0}^{k-1} H_{i+l, j+l}(s, c, g)\right\} \mathbf{P}\left\{X_{i+k+s-1} \neq Y_{j+k+s-1}\right\} \\
& =C_{s-2 g-k+1}^{c-2 g-k+1}(1-R)^{s-c+2} R^{c+r-1} .
\end{aligned}
$$

Из нее следует, что

$$
\begin{aligned}
\sum_{a \in \Gamma} \mathbf{E} W(a) & =\sum_{i=1}^{m} \sum_{j=1}^{n} \sum_{k=1}^{h} \mathbf{E} W(i, j, k) \leqslant C_{s-2 g}^{s-c}(1-R)^{s-c+2} m n \sum_{k=1}^{h} R^{c+k-1} \\
& <m n C_{s-2 g}^{s-c}(1-R)^{s-c+1} R^{c}=\Lambda .
\end{aligned}
$$

Значит,

$$
\begin{aligned}
\mathbf{E} W(a)+\mathbf{E} U(a) & \leqslant \sum_{r=1}^{h}\left|\left(\{a\} \cup \Gamma_{a}\right) \cap \Gamma(r)\right| C_{s-2 g-r+1}^{s-c}(1-R)^{s-c+2} R^{c+r-1} \\
& \leqslant C_{s-2 g}^{s-c}(2(s+h)+3)(m+n)(1-R)^{s-c+2} \sum_{r=1}^{h} R^{c+r-1} \\
& \leqslant C_{s-2 g}^{s-c}(2(s+h)+3)(m+n)(1-R)^{s-c+1} R^{c} \\
& =\Lambda(2(s+h)+3)\left(m^{-1}+n^{-1}\right) .
\end{aligned}
$$

Поэтому

$$
\sum_{a \in \Gamma} \mathbf{E} W(a)(\mathbf{E} W(a)+\mathbf{E} U(a)) \leqslant(2(s+h)+3)\left(m^{-1}+n^{-1}\right) \Lambda^{2} .
$$

Оценим вторую сумму в правой части (2.11). Заметим, что для элементов множества $\Gamma_{a}$ выполнено одно из условий (или выполнены оба условия сразу)

$$
-\left(s+k^{\prime}\right) \leqslant i^{\prime}-i \leqslant s+k, \quad-\left(s+k^{\prime}\right) \leqslant j^{\prime}-j \leqslant s+k .
$$


Рассмотрим отдельно разные варианты. Введем множества

$$
\begin{aligned}
& \Gamma_{a}^{\text {str }}=\left\{a^{\prime}=\left(i^{\prime}, j^{\prime}, k^{\prime}\right) \in \Gamma_{a}:-\left(s+k^{\prime}\right) \leqslant i^{\prime}-i=j^{\prime}-j \leqslant s+k\right\}, \\
& \Gamma_{a}^{*}=\Gamma_{a} \backslash \Gamma_{a}^{\mathrm{str}}, \\
& \Gamma_{a}^{* *}=\left\{a^{\prime} \in \Gamma_{a}^{*}: 0<i^{\prime}-i \leqslant s+k \text { или } 0<i-i^{\prime} \leqslant s+k^{\prime},\right. \\
&\left.\quad 0<j^{\prime}-j \leqslant s+k \text { или } 0<j-j^{\prime} \leqslant s+k^{\prime}\right\} .
\end{aligned}
$$

Для элементов множеств $\Gamma_{a}^{\text {str }}$ и $\Gamma_{a}^{* *}$ выполнены оба условия (2.14).

Если $a^{\prime} \in \Gamma_{a}^{*} \backslash \Gamma_{a}^{* *}$, то выполнено лишь одно из условий (2.14). Нам удобно рассмотреть два имеющихся варианта отдельно. Определим множества

$$
\begin{aligned}
& \Gamma_{a, 1}^{* \mid * *}=\left\{a^{\prime} \in \Gamma_{a}^{*} \backslash \Gamma_{a}^{* *}: 0<i^{\prime}-i \leqslant s+k \text { или } 0<i-i^{\prime} \leqslant s+k^{\prime}\right\}, \\
& \Gamma_{a, 2}^{* * * *}=\left\{a^{\prime} \in \Gamma_{a}^{*} \backslash \Gamma_{a}^{* *}: 0<j^{\prime}-j \leqslant s+k \text { или } 0<j-j^{\prime} \leqslant s+k^{\prime}\right\} .
\end{aligned}
$$

Тогда

$$
\Gamma_{a, 1}^{* \backslash * *} \cap \Gamma_{a, 2}^{* \backslash * *}=\varnothing \quad \text { и } \quad \Gamma_{a}^{*} \backslash \Gamma_{a}^{* *}=\Gamma_{a, 1}^{* \backslash * *} \cup \Gamma_{a, 2}^{* \backslash * *} .
$$

Заметим, что

$$
\Gamma_{a} \backslash\{a\}=\Gamma_{a}^{\mathrm{str}} \cup \Gamma_{a, 1}^{* \backslash * *} \cup \Gamma_{a, 2}^{* \backslash * *} \cup \Gamma_{a}^{* *} .
$$

Лемма 3. Пусть $a \in \Gamma(k), a^{\prime} \in \Gamma_{a}^{\mathrm{str}} \cap \Gamma\left(k^{\prime}\right), k, k^{\prime} \leqslant h$.

(a) Eсли $i=i^{\prime}, j=j^{\prime}, k \neq k^{\prime}$, mo $\mathbf{E} W(a) W\left(a^{\prime}\right)=0$.

(б) Если $0<i^{\prime}-i \leqslant g+k-1$ или $0<i-i^{\prime} \leqslant g+k^{\prime}-1$, то $\mathbf{E} W(a) W\left(a^{\prime}\right)=0$.

(в) $E c л и g+k \leqslant i^{\prime}-i \leqslant s+k$ или $g+k^{\prime} \leqslant i-i^{\prime} \leqslant s+k^{\prime}$, mo $\mathbf{E} W(a) W\left(a^{\prime}\right) \leqslant R^{g+k^{\prime}-1} \mathbf{E} W(a)$.

Доказательство леммы 3 . Воспользуемся вытекающим из определений соотношением

$$
\begin{aligned}
G(i, j, k) \subseteq & \bigcap_{t=0}^{g+k-2}\left\{X_{i+t}=Y_{j+t}\right\} \cap \bigcap_{t=0}^{k-1}\left\{X_{i+s+t-1}=Y_{j+s+t-1}\right\} \\
& \bigcap\left\{X_{i-1} \neq Y_{j-1}\right\} \cap\left\{X_{i+s+k-1} \neq Y_{j+s+k-1}\right\} .
\end{aligned}
$$

Аналогично,

$$
\begin{aligned}
G\left(i^{\prime}, j^{\prime}, k^{\prime}\right) \subseteq & \bigcap_{t=0}^{g+k^{\prime}-2}\left\{X_{i^{\prime}+t}=Y_{j^{\prime}+t}\right\} \cap \bigcap_{t=0}^{k^{\prime}-1}\left\{X_{i^{\prime}+s+t-1}=Y_{j^{\prime}+s+t-1}\right\} \\
& \bigcap\left\{X_{i^{\prime}-1} \neq Y_{j^{\prime}-1}\right\} \cap\left\{X_{i^{\prime}+s+k^{\prime}-1} \neq Y_{j^{\prime}+s+k^{\prime}-1}\right\} .
\end{aligned}
$$

Докажем утверждение (а). Пусть $i=i^{\prime}, j=j^{\prime}, k>k^{\prime}$. Тогда, согласно (2.16) и (2.17),

$$
\begin{aligned}
& G(i, j, k) \subseteq\left\{X_{i^{\prime}+s+k^{\prime}-1}=Y_{j^{\prime}+s+k^{\prime}-1}\right\}, \\
& G\left(i^{\prime}, j^{\prime}, k^{\prime}\right) \subseteq\left\{X_{i^{\prime}+s+k^{\prime}-1} \neq Y_{j^{\prime}+s+k^{\prime}-1}\right\} .
\end{aligned}
$$


Значит, события $G(i, j, k)$ и $G\left(i^{\prime}, j^{\prime}, k^{\prime}\right)$ несовместны и

$$
\mathbf{E} W(a) W\left(a^{\prime}\right)=\mathbf{P}\left\{G_{k}(i, j, k) \cap G\left(i^{\prime}, j^{\prime}, k^{\prime}\right)\right\}=0 .
$$

Точно так же обстоит дело при $i=i^{\prime}, j=j^{\prime}, k>k^{\prime}$. Утверждение (а) доказано.

Докажем утверждение (б). Пусть $0<i^{\prime}-i \leqslant g+k-1$. Из (2.16) и $(2.17)$ следует, что

$$
G(i, j, k) \subseteq\left\{X_{i^{\prime}-1}=Y_{j^{\prime}-1}\right\}, \quad G\left(i^{\prime}, j^{\prime}, k^{\prime}\right) \subseteq\left\{X_{i^{\prime}-1} \neq Y_{j^{\prime}-1}\right\} .
$$

Значит, и в этом случае события $G(i, j, k)$ и $G\left(i^{\prime}, j^{\prime}, k^{\prime}\right)$ несовместны, а

$$
\mathbf{E} W(a) W\left(a^{\prime}\right)=\mathbf{P}\left\{G(i, j, k) \cap G\left(i^{\prime}, j^{\prime}, k^{\prime}\right)\right\}=0 .
$$

Точно так же обстоит дело при $0<i-i^{\prime} \leqslant g+k^{\prime}-1$. Утверждение (б) доказано.

Докажем первую часть утверждения (в). В этом случае

$$
G\left(i^{\prime}, j^{\prime}, k^{\prime}\right) \subseteq \bigcap_{t=0}^{g+k^{\prime}-2}\left\{X_{i^{\prime}+s-g+t}=Y_{j^{\prime}+s-g+t}\right\},
$$

причем $i^{\prime}+s-g>i+s+k-1$. Значит, события $G(i, j, k)$ и $\bigcap_{t=0}^{g+k^{\prime}-2}\left\{X_{i^{\prime}+s-g+t}=Y_{j^{\prime}+s-g+t}\right\}$ независимы и

$$
\begin{aligned}
\mathbf{E} W(a) W\left(a^{\prime}\right) & \leqslant \mathbf{P}\left\{G_{k}(i, j, k)\right\} \mathbf{P}\left\{\bigcap_{t=0}^{g+k^{\prime}-2}\left\{X_{i^{\prime}+s-g+t}=Y_{j^{\prime}+s-g+t}\right\}\right\} \\
& =\mathbf{E} W(a) R^{g+k^{\prime}-1} .
\end{aligned}
$$

Первая часть утверждения (в) доказана. Вторая часть этого утверждения доказывается аналогично. Доказательство леммы 3 завершено.

Лемма 4. Пусть $a \in \Gamma$. Тогда:

(a) если $a^{\prime} \in \Gamma_{a, 1}^{* * * *}$, mo $\mathbf{E} W(a) W\left(a^{\prime}\right)<C_{s}^{d} q^{c} \mathbf{E} W(a)$,

(б) если $a^{\prime} \in \Gamma_{a, 2}^{* * * *}$, mo $\mathbf{E} W(a) W\left(a^{\prime}\right)<C_{s}^{d} p^{c} \mathbf{E} W(a)$,

(в) если $a^{\prime} \in \Gamma_{a}^{* *}$, mo

$$
\mathbf{E} W(a) W\left(a^{\prime}\right)<\left(C_{s}^{d}\right)^{2} r^{c} .
$$

При доказательстве леммы 4 мы во многом следуем доказательству утверждений (b) и (c) леммы 1 работы [6].

(a) Пусть $a^{\prime} \in \Gamma_{a, 1}^{* * *}$. Имеем

$$
\begin{aligned}
& \mathbf{E} W(a) W\left(a^{\prime}\right)= \sum_{x_{-1}, \ldots, x_{s+k^{\prime}-1}} \mathbf{E}\left(W(a) W\left(a^{\prime}\right) I\left\{A\left(x_{-1}, \ldots, x_{s+k^{\prime}-1}\right)\right\}\right) \\
&=\sum_{x_{-1}, \ldots, x_{s+k^{\prime}-1}}\left(\mathbf{E}\left(W\left(a^{\prime}\right) \mid A\left(x_{-1}, \ldots, x_{s+k^{\prime}-1}\right) \cap G(i, j, k)\right)\right. \\
&\left.\times \mathbf{P}\left\{G(i, j, k) \cap A\left(x_{-1}, \ldots, x_{s+k^{\prime}-1}\right)\right\}\right),
\end{aligned}
$$

где

$$
A\left(x_{-1}, \ldots, x_{s+k^{\prime}-1}\right)=\left\{X_{i^{\prime}-1}=x_{-1}, \ldots, X_{i^{\prime}+s+k^{\prime}-1}=x_{s+k^{\prime}-1}\right\} .
$$


Так как множества $\{j-1, j, \ldots, j+s+k-1\}$ и $\left\{j^{\prime}-1, j^{\prime}, \ldots, j^{\prime}+s+k^{\prime}-1\right\}$ не пересекаются, то в тех случаях, когда

$$
\mathbf{P}\left\{A\left(x_{-1}, \ldots, x_{s+k^{\prime}-1}\right) \cap G(i, j, k)\right\}>0,
$$

выполнены соотношения

$$
\begin{aligned}
& \mathbf{E}\left(W\left(a^{\prime}\right) \mid A\left(x_{-1}, \ldots, x_{s+k^{\prime}-1}\right) \cap G(i, j, k)\right) \\
& \quad=\mathbf{E}\left(W\left(a^{\prime}\right) \mid A\left(x_{-1}, \ldots, x_{s+k^{\prime}-1}\right)\right) \leqslant C_{s-k-2 g}^{d} q^{c}<C_{s}^{d} q^{c},
\end{aligned}
$$

где в качестве числового коэффициента первый раз фигурирует число вариантов расположения «несовпадений» букв в цепочках, а второй раз его оценка сверху. В итоге получаем, что

$$
\begin{aligned}
\mathbf{E} W(a) W\left(a^{\prime}\right) & <C_{s}^{d} q^{c} \sum_{x_{-1}, \ldots, x_{s+k^{\prime}-1}} \mathbf{P}\left\{G(i, j, k) \cap A\left(x_{-1}, \ldots, x_{s+k^{\prime}-1}\right)\right\} \\
& =C_{s}^{d} q^{c} \mathbf{P}\{G(i, j, k)\}=C_{s}^{s-c} q^{c} \mathbf{E} W(a) .
\end{aligned}
$$

Таким образом, утверждение (а) доказано.

Утверждение (б) доказывается аналогично.

(в) Пусть $a^{\prime} \in \Gamma_{a}^{* *}$. Рассмотрим множество

$$
\Delta_{g}=\left\{\delta=\left(\delta_{1}, \ldots, \delta_{s}\right) \in\{0,1\}^{s}: \sum_{k=1}^{s} \delta_{k}=c, \delta_{1}=\cdots=\delta_{g}=\delta_{s}=1\right\} .
$$

При $\delta \in \Delta_{g}$ положим

$$
H_{i, j, \delta}=\left\{X_{i+t-1}=Y_{j+t-1} \Longleftrightarrow \delta_{t}=1, t=1, \ldots, s\right\} .
$$

Рассмотрим случайные величины

$$
W(a, \delta)=W(a) I\left\{H_{i, j, \delta}\right\}, \quad a=(i, j, k), \quad \delta \in \Delta_{g} .
$$

Почти дословно повторив рассуждения доказательства утверждения (c) леммы 1 работы [6], получим, что

$$
\mathbf{E} W(a, \delta) W\left(a^{\prime}, \delta^{\prime}\right) \leqslant r^{c} .
$$

Заметим теперь, что

$$
W(a)=\sum_{\delta \in \Delta_{g}} W(a, \delta), \quad\left|\Delta_{g}\right|<C_{s}^{d} .
$$

Поэтому из (2.19) следует неравенство (2.18). Лемма 4 доказана.

Продолжим доказательство леммы 2. Заметим, что, согласно формулам (2.1) и (2.12),

$$
\sum_{a \in \Gamma} \mathbf{E} W(a)=\sum_{k=1}^{h} \sum_{a \in \Gamma(k)} \mathbf{E} W(a)<\Lambda .
$$

Кроме того,

$$
\begin{gathered}
\left|\Gamma_{a}^{\operatorname{str}} \cap \Gamma\left(k^{\prime}\right)\right|<2 s, \quad\left|\Gamma_{a}^{* *}\right| \leqslant 4(s+h)^{2}, \\
\left|\Gamma_{a, 1}^{* \backslash * *}\right|<2(s+h) n, \quad\left|\Gamma_{a, 2}^{* \backslash * *}\right|<2(s+h) m .
\end{gathered}
$$


Поэтому, используя леммы 3 и 4, получаем

$$
\begin{aligned}
& \sum_{a \in \Gamma} \sum_{a^{\prime} \in \Gamma_{a}^{\text {str }}} \mathbf{E} W(a) W\left(a^{\prime}\right)=\sum_{a \in \Gamma} \sum_{k^{\prime}=1}^{h} \sum_{a^{\prime} \in \Gamma_{a}^{\text {str }} \cap \Gamma\left(k^{\prime}\right)} \mathbf{E} W(a) W\left(a^{\prime}\right) \\
& \leqslant \sum_{a \in \Gamma} \mathbf{E} W(a) \sum_{k^{\prime}=1}^{h}\left|\Gamma_{a}^{\mathrm{str}} \cap \Gamma\left(k^{\prime}\right)\right| R^{g+k^{\prime}-1}<2(s+h) R^{g} \Lambda(1-R)^{-1}, \\
& \sum_{a \in \Gamma} \sum_{a^{\prime} \in \Gamma_{a, 1}^{*} \backslash * *} \mathbf{E} W(a) W\left(a^{\prime}\right)<2(s+h) C_{s}^{d} n q^{c} \Lambda, \\
& \sum_{a \in \Gamma} \sum_{a^{\prime} \in \Gamma_{a, 2}^{* \backslash \backslash * *}} \mathbf{E} W(a) W\left(a^{\prime}\right)<2(s+h) C_{s}^{d} m p^{c} \Lambda, \\
& \sum_{a \in \Gamma} \sum_{a^{\prime} \in \Gamma_{a}^{* *}} \mathbf{E} W(a) W\left(a^{\prime}\right) \leqslant 4(s+h)^{2}\left(C_{s}^{d}\right)^{2} m n r^{c} .
\end{aligned}
$$

В силу (2.15) и неравенства $h \leqslant s$ из этих оценок получаем:

$$
\begin{aligned}
& \sum_{a \in \Gamma} \sum_{a^{\prime} \in \Gamma_{a}} \operatorname{EW}(a) W\left(a^{\prime}\right) \\
& \quad<4 s \Lambda\left(R^{g}(1-R)^{-1}+C_{s}^{d}\left(m p^{c}+n q^{c}\right)\right)+16 s^{2}\left(C_{s}^{d}\right)^{2} m n r^{c}
\end{aligned}
$$

Подставим эту оценку вместе с оценкой (2.13) в неравенство (2.11) и после несложных преобразований получим неравенство (2.7). Лемма 2 доказана.

Пусть $\pi_{1}, \ldots, \pi_{h}$ - независимые случайные величины, распределенные по закону Пуассона с параметрами

$$
\lambda_{k}(m, n, c)=\Lambda R^{k-1}(1-R), \quad k=1, \ldots, h,
$$

соответственно. Рассмотрим случайную величину

$$
\pi(h)=\sum_{k=1}^{h} k \pi_{k}
$$

Следствие 1. Пусть $m, n>s=c+d \geqslant c \geqslant 2 g$. Тогда

$$
\rho\left(\mathscr{L}\left(N_{d}(g)\right), \mathscr{L}(\pi(h))\right) \leqslant S_{1}+S_{2} .
$$

Д ок азатель с т в о сл ед с т в я 1. Для произвольной функции $F$ на множестве $\{0,1,2, \ldots\}^{\Gamma}$ выполнено неравенство

$$
\rho(\mathscr{L}(F(W)), \mathscr{L}(F(\pi))) \leqslant \rho(\mathscr{L}(W), \mathscr{L}(\pi)) .
$$

Возьмем функцию

$$
F(x)=\sum_{k=1}^{h} k \sum_{i=1}^{m} \sum_{j=1}^{n} x_{i, j, k}, \quad k=1, \ldots, h,
$$

где $x=\left(x_{i, j, k},(i, j, k) \in \Gamma\right)$. Тогда, согласно формулам (2.6) и (2.20),

$$
N_{d}(g)=F(W), \quad \mathscr{L}(\pi(h))=\mathscr{L}(F(\pi)) .
$$


Поэтому из неравенств (2.7) и (2.22) вытекает неравенство (2.21). Следствие 1 доказано.

Доказательств о те о ремы 1. Положим

$$
g=\left[\frac{\ln s+\ln \ln s}{|\ln R|}\right]+1 \text {. }
$$

Тогда

$$
s R^{g} \leqslant s R^{(\ln s+\ln \ln s) /|\ln R|}=\frac{1}{\ln s} \longrightarrow 0 .
$$

Поэтому из условия (1.6) (и, тем более, из условия (1.2) теоремы 1) вытекает соотношение $S_{2} \rightarrow 0$. Кроме этого, непосредственно из условий теоремы следуют соотношения

$$
\frac{d g}{s} \rightarrow 0, \quad S_{1} \rightarrow 0 .
$$

Поэтому можно воспользоваться леммой 1 и следствием 1 , согласно которым

$$
\mathbf{P}\left\{N_{d}=N_{d}(g)\right\} \longrightarrow 1, \quad \rho\left(\mathscr{L}\left(N_{d}(g)\right), \mathscr{L}(\pi(h))\right) \longrightarrow 0 .
$$

Заметим, что в данном случае $h=c-2 g+1 \rightarrow \infty$, a

$$
\lambda_{k}(m, n, c) \rightarrow \lambda \rho^{k-1}(1-\rho), \quad k=1,2, \ldots .
$$

Значит, распределение случайной величины $\pi(h)$ сходится к распределению с производящей функцией (1.5). Согласно (2.23), к нему же сходится и распределение случайной величины $N_{d}$. Теорема 1 доказана.

3 а м е ч а н и е 9. Использованный в настоящей работе метод позволяет получать и оценки точности аппроксимации распределения случайной величины $N_{d}$ сопровождающим и предельным сложными пуассоновскими распределениями. При доказательстве теоремы 1 выведены некоторые такие оценки для вспомогательных случайных величин (лемма 2 и следствие 1). Для получения оценок точности аппроксимации может быть использован также метод работы [10]. Этим методом в работах [6] и [11] были получены оценки точности аппроксимации распределения случайной величины $N_{0}$ (в [6]) и распределений случайных величин $N_{d}$ (в [11]) сопровождающими сложными пуассоновскими распределениями, построенными по рекомендациям работы [10]. Однако для случая $d>0$ явных выражений для параметров сопровождающего распределения из-за его громоздкости получено не было. В работе [6] было показано, что рассматриваемое там сопровождающее сложное пуассоновское распределение сходится к распределению с производящей функцией $(1.5)$.

Автор признателен Н. М. Меженной и рецензенту за полезные замечания. 


\section{СПИСОК ЛИТЕРАТУРЫ}

1. Arratia R., Gordon L., Waterman M.S. An extreme value theory for sequence matching. - Ann. Statist., 1986, v. 14, № 3, p. 971-993.

2. Arratia R., Waterman M.S. The Erdös-Rényi strong law for pattern matching with a given proportion of mismatches. - Ann. Probab., 1989, v. 17, № 3, p. 1152-1169.

3. Arratia R., Gordon L., Waterman M.S. The Erdös-Rényi law in distribution, for coin tossing and sequence matching. - Ann. Statist., 1990, v. 18, № 2, p. 539-570.

4. Новак С. Ю. Пуассонова аппроксимация числа длинных «повторов» в случайных последовательностях. - Теория вероятн. и ее примен., 1994, т. 39, в. 4, с. 731-742; 1995 , т. 40 , в. 3 , с. 700 .

5. Novak S. Yu. Long match patterns in random sequences. - Siberian Adv. Math., 1995 , v. 5, № 3, p. 128-140.

6. Михайлов В.Г. Оценка точности сложной пуассоновской аппроксимации для распределения числа совпадаюших цепочек. - Теория вероятн. и ее примен., 2001, т. 46 , в. 4 , с. $713-723$.

7. Михайлов В.Г. Предельные теоремы пуассоновского типа для числа неполных совпадений $s$-цепочек. - Теория вероятн. и ее примен., 2002, т. 47 , в. 2, с. 350357.

8. Зубков A.М., Михайлов В.Г. Предельные распределения случайных величин, связанных с длинными повторениями в последовательности независимых испытаний. - Теория вероятн. и ее примен., 1974, т. 19, в. 1, с. 173-181.

9. Зубков А. М., Михайлов В. Г. О повторениях $s$-цепочек в последовательности независимых величин. - Теория вероятн. и ее примен., 1979, т. 24, в. 2, с. 267-279.

10. Roos $M$. Stein's method for compound Poisson approximation: the local approach. Ann. Appl. Probab., 1994, v. 4, p. 1177-1187.

11. Månsson $M$. On compound Poisson approximation for sequence matching. - Combin., Probab. Comp., 2000, v. 9, №6, p. 529-548.

12. Barbour A. D., Holst L., Janson S. Poisson Approximation. New York: Oxford Univ. Press, 1992, $277 \mathrm{p}$.

Поступила в редакцию

12.XII. 2005

Исправленный вариант 15.V.2007 